\title{
Assembly-Induced Emission and Stability Enhancement of Cu Nanoparticles
}

\author{
Tingyao Zhou and Jinbin Liu* \\ Key Laboratory of Functional Molecular Engineering of Guangdong Province, School of Chemistry and \\ Chemical Engineering, South China University of Technology, Guangzhou, Guangdong 510640, China
}

Email: cejbliu@scut.edu.cn (J. L.)

\begin{abstract}
The exploitation of luminescent Cu nanoparticles (CuNPs) with excellent stability and bright emission is highly desired but still challenging. In this perspective, we concern the significance of aggregation-induced emission (AIE) effect for designing highly luminescent CuNPs, and highlight recent advances for enhancement of stability and emission of the CuNPs using self-assembly approach. Finally, we discuss perspectives and research directions for the fabrication of the highly luminescent assembled CuNPs in the future.
\end{abstract}

Keywords self-assembly, block copolymers, luminescence enhancement, Cu nanoparticles

The few-nanometer luminescent metal nanoparticles (NPs, $d<3 \mathrm{~nm}$ ), bridging the gap between metal atom and plasmatic NPs, have received extensive attention in the past few decades for their unique physicochemical properties and great potential applications in chemical sensor, catalysis, electronic devices, bioimaging and therapy. ${ }^{[1]}$ To date, a variety of luminescent metal NPs have been exploited, such as ultrasmall AuNPs, AgNPs, CuNPs, PtNPs, as well as alloy NPs. ${ }^{[2]}$ Among these, ultrasmall CuNPs are of immense interest due to the earth abundance and rather inexpensive price of copper, ${ }^{[3]}$ and various synthetic approaches, including chemical reduction, chemical etching, electrochemical and photochemical synthesis, have been developed for preparing luminescent CuNPs in the presence of capping agents. However, the luminescent CuNPs usually suffered from instability and were inclined to form large non-luminescent particles or small molecular copper complexes because of the easy surface oxidation of copper upon exposure to air, ${ }^{[4]}$ raising a new challenge for their universal applications. It is highly desirable to explore a facile strategy for achieving excellently stable and brightly emitting CuNPs.

Aggregation-induced emission (AIE) enhancement provided a new avenue for designing novel photoluminescent materials. ${ }^{[5]}$ The AIE mechanism was derived from the restriction of intramolecular vibrations and rotations and the enhancement of radiative transition delay in the aggregation state. Inspired from AIE effect, a dramatic growth has been witnessed in developing highly luminescent CuNP-based materials. The aggregation of luminescent CuNP was formed to improve the stability and emission through changing microenvironment, such as $\mathrm{pH}$, solvent or addition of new ions. $^{[6,7]}$ For example, Wang' group reported ultrasmall CuNPs could generate an obvious luminescence enhancement upon aggregation by addition of ethanol, and obtained large CuNP aggregates were stable for several months without an inert gas protection. ${ }^{[7]}$ The stability enhancement was attributed to the decrease of specific surface area in comparison to the individual CuNP.

Self-assembly approach was particularly attractive for arranging metal NPs into well-controlled and ordered nanostructures, ${ }^{[8]}$ which could endow the instable weak-emitting CuNPs with enhanced stability and emission. Recently, two types of highly compact and ordered nanoarchitectures, namely nanoribbons and nanosheets, were self-assembled using 1-dodecanethiol (DT)-capped CuNPs as building blocks, which could enhance luminescent performance of ultrasmall CuNPs through intensifying the cuprophilic $\mathrm{Cu}(\mathrm{I}) \cdots \mathrm{Cu}(\mathrm{I})$ interactions of NPs and restraining intra-molecular rotation and vibration of the surface capping ligands, and also tune emission color of formed assemblies by controlling the regularity of NPs. ${ }^{[9]}$ Using the similar luminescent CuNPs as a model, ethanol was introduced during self-assembly process, and resulted in rapid generation of ultrathin nanosheets with metal defects-rich surface, improving their emission performance greatly. ${ }^{[10]}$ Moreover, other copper-based self-assembly architectures, such as nanowires and thin films, ${ }^{[12,13]}$ were also prepared by regulating the multiple intermolecular interactions to realize assemblyinduced emission enhancement of CuNPs. For example, Wang et al. employed hypromellose grafted chitosan as triggering agent for CuNPs aggregation and fabricated a luminescent CUNP composite film with AIE enhancement, which led to quantum yield as high as $42 \%$. The composite film could be utilized as color converters for preparation of remote light-emitting devices. ${ }^{[12]}$

An alternative approach has been exploited using amphiphilic block copolymer as templates to produce well-defined assembled nanoarchitectures in aqueous solution. ${ }^{[13]}$ Amphiphilic block copolymer could self-assemble and form controllable aggregates with different morphologies by altering copolymer composition or reaction parameters, providing a promising template for the arrangement of NPs. The previous efforts focused mainly on assembling pre-synthesized and stable large metal NPs or semiconducting NPs using functionalized amphiphilic block copolymer with sophisticated synthesis process. ${ }^{[14]}$ Most recently, our group demonstrated a facile and extensible approach for in-situ preparation of highly stable and luminescent $\mathrm{Cu}$ nanoassemblies with encapsulated CuNP number control using commercially available amphiphilic triblock copolymer as templates (Figure 1). ${ }^{[15]}$ Taking the advantages of tunable size of triblock copolymer templates, the number of encapsulated CuNP inside an formed assembly could be greatly controllable by regulating the template block segments. Both the stability and emitting brightness of the $\mathrm{Cu}$ nanoassemblies were greatly enhanced in the complicated physiological conditions using a multi-dentate thiol ligand as cross-linking agent, even in the lysosomal acidic microenvironment with $\mathrm{pH}$ as low as $\sim 4.5$, the fabricated $\mathrm{Cu}$ 


\section{Perspective}

nanoassemblies as a potential optical nanoprobe could remain brightly stable for $24 \mathrm{~h}$. This work opened a feasible and useful guidance for preparing stable and controllable assemblies using instable metal NPs.

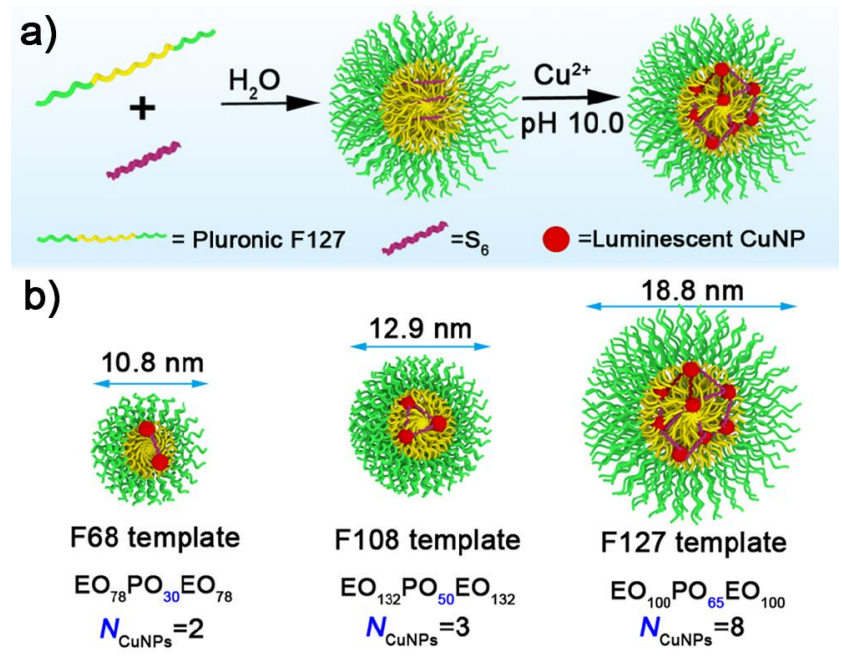

Figure 1 (a) Schematic illustration of in-situ synthesis of luminescent $\mathrm{Cu}$ nanoassemblies templated form pluronic F127. (b) Schematic illustration of the $\mathrm{Cu}$ nanoassemblies with encapsulated CuNP number control through regulating the template block segments. Reprinted with permission from Ref. [15]. Copyright 2019 American Chemical Society.

In summary, achieving highly stable and brightly emitting CuNPs is fascinating but still challenging. The AIE effect can enhance the stability and emission of ultrasmall CuNPs through restricting intramolecular vibrations and rotations and reducing the specific surface area upon aggregation. Self-assembly strategy is undoubtedly an effective approach for designing well-controlled and ordered luminescent copper- based nanostructures. With the assistance of advantages of amphiphilic block copolymer templates and cross-linking interaction, the well-defined and controllable $\mathrm{Cu}$ nanoassemblies could still remain high stability and bright emission in physiological environments, which would stimulate more downstream biomedical applications based on luminescent CuNPs. Though progresses have been made, more great efforts should be required for this field. By controlling the size and shape of block copolymers templates, the $\mathrm{Cu}$ nanoassemblies with versatile morphologies could be in-situ prepared and exploited for specific applications. Through hybridization of other functional materials, such as drugs and probes, or surface functionalization with targeting reagents, the construction of various uniform nanomaterials with high stability and desired functions could be easily designed for highly sensitive chemical sensor and biological applications. With the continuous progress of the cross discipline between chemistry, biology, and medicine, we foresee biological stable and bright CuNP-based nanomedicines will be designed for cancer diagnosis and therapy or to even greater heights in the future.

\section{Acknowledgement}

This work was supported by the National Natural Science Foundation of China (Grant No. 21573078), the Guangdong Natural Science Funds for Distinguished Young Scholars (Grant No. 2016A030306024), the Natural Science Foundation of Guangdong Province (Grant No. 2018A030310376), the China Postdoctoral Science Foundation (Grant No. 2017M622672), and the Fundamental Research Funds for the Central Universities.

\section{References}

[1] Jin, R.; Zeng, C.; Zhou, M.; Chen, Y. Chem. Rev. 2016, 116, 10346.

[2] Chakraborty, I.; Pradeep, T. Chem. Rev. 2017, 117, 8208.

[3] Liu, X.; Astruc, D. Coord. Chem. Rev. 2018, 359, 112.

[4] Yuan, P.; Chen, R.; Zhang, X.; Chen, F.; Yan, J.; Sun, C.; Ou, D.; Peng, J.; Lin, S.; Tang, Z.; Teo, B. K.; Zheng, L. S.; Zheng, N. Angew. Chem. Int. Ed. 2019, 58, 835.

[5] Goswami, N.; Yao, Q.; Luo, Z.; Li, J.; Chen, T.; Xie, J. J. Phys. Chem. Lett. 2016, 7, 962.

[6] Jia, X.; Yang, X.; Li, J.; Li, D.; Wang, E. Chem. Commun. 2014, 50, 237.

[7] Jia, X.; Li, J.; Wang, E. Small 2013, 9, 3873.

[8] Boles, M. A.; Engel, M.; Talapin, D. V. Chem. Rev. 2016, 116, 11220.

[9] Wu, Z.; Liu, J.; Gao, Y.; Liu, H.; Li, T.; Zou, H.; Wang, Z.; Zhang, K.; Wang, Y.; Zhang, H.; Yang, B. J. Am. Chem. Soc. 2015, 137, 12906.

[10] Wu, Z.; Liu, H.; Li, T.; Liu, J.; Yin, J.; Mohammed, O. F.; Bakr, O. M. Liu, Y.; Yang, B.; Zhang, H. J. Am. Chem. Soc. 2017, 139, 4318.

[11] Wu, Z.; Li, Y.; Liu, J.; Lu, Z.; Zhang, H.; Yang, B. Angew. Chem. Int Ed. 2014, 53, 12196.

[12] Wang, Z. G.; Shi, Y. E.; Yang, X. M.; Xiong, Y.; Li, Y. X.; Chen, B. K.; Lai, W. F.; Rogach, A. L. Adv. Funct. Mater. 2018, 28, 1802848.

[13] Mai, Y.; Eisenberg, A. Acc. Chem. Res. 2012, 45, 1657.

[14] Hu, J.; Wu, T.; Zhang, G.; Liu, S. J. Am. Chem. Soc. 2012, 134, 7624.

[15] Zhou, T.; Zhu, J.; Gong, L.; Nong, L.; Liu, J. J. Am. Chem. Soc. 2019, 141, 2852.

Received February 21, 2019

Accepted March 16, 2019 